\title{
Erratum: One-pion-exchange effect in the energy spectrum of muonic hydrogen [Phys. Rev. A 92, 032512 (2015)]
}

\author{
Hai-Qing Zhou and Hou-Rong Pang
}

(Received 31 May 2016; published 13 June 2016)

DOI: 10.1103/PhysRevA.93.069903

There is a mistake in the last term of Eq. (9) we used in the paper, the correct form should be read as

$$
V_{l p}^{\pi^{0}}(r)=\frac{c_{1, l} g_{\pi^{0} \gamma \gamma} f_{\pi N N}}{12 \pi}\left\{\left[\left(3 \widehat{r} \cdot \vec{\sigma}_{1} \widehat{r} \cdot \vec{\sigma}_{2}-\vec{\sigma}_{1} \cdot \vec{\sigma}_{2}\right)\left(1+\frac{3}{M_{\pi} r}+\frac{3}{M_{\pi}^{2} r^{2}}\right)+\vec{\sigma}_{1} \cdot \vec{\sigma}_{2}\right] \frac{e^{-M_{\pi} r}}{r}-\frac{4 \pi}{M_{\pi}^{2}} \vec{\sigma}_{1} \cdot \vec{\sigma}_{2} \delta^{3}(\vec{r})\right\} .
$$

After this correction, the numbers 0.0028 and $1.161 \times 10^{-9} \mathrm{meV}$ in the paper should be corrected as -0.057 and $-1.77 \times$ $10^{-10} \mu \mathrm{eV}$. And Fig. 4 should be corrected as given below.

This correction will not change the main conclusion of this paper: The contribution from the one-pion exchange is small, and the contribution from the scalar meson exchange may play a more important role.

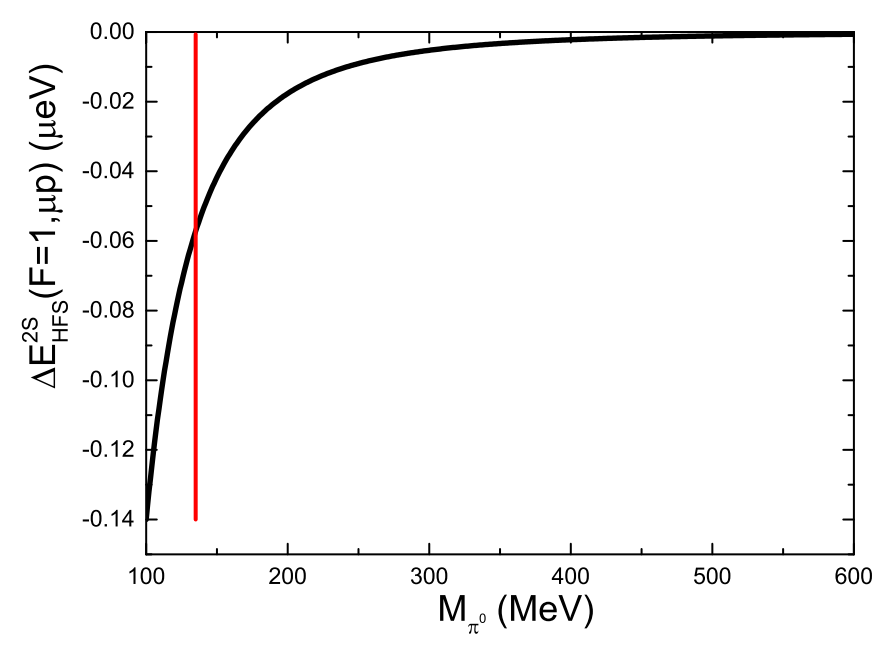

FIG. 4. The mass dependence of the contribution to $\Delta E_{\mathrm{HFS}}^{2 S}(F=1, \mu p)$. 